\title{
Enhancement of Online Computerized Examination by Incorporating Artificial Intelligence to One Word Answer Questions Along with Multiple Choice Questions
}

\author{
Vimal P. Parmar \\ Research Scholar, \\ Dept. of Comp. Sci. \\ Saurashtra University, Rajkot. Gujarat, INDIA
}

\author{
C.K. Kumbharana, PhD \\ Head, Guide \\ Department of Computer Science \\ Saurashtra University Rajkot. \\ Gujarat, INDIA
}

\begin{abstract}
Today due to the increased number of various courses and increased number of appearing students, the process of examination has become more complex. Many hours of examiners and human efforts are required for effective evaluation of such examination. Computer is a powerful tool that can be used as a solution for such a complex problem. Many educational institutes, software industries, organizations, colleges, departments and universities have employed computerized on-line examinations which consisting of multiple choice questions that does not only perform the error free evaluation but also generates the fast result. It is a good solution than none but what about the evaluation of subjective type theoretical examination in which examiners have to decide and assign marks to the answers. To design such an intelligence system that automatically evaluates subjective type question answers require tremendous efforts including building of knowledge base for various subjects' knowledge. Recently computer is widely used for online examination but in most of the cases only objective type multiple choice questions are involved. It is easier for a computer to evaluate objective type multiple choice questions but it is too much difficult for a computer to evaluate subjective type questions as similar as intelligent human efforts require.
\end{abstract}

In this research paper here the effort is being made for the one word answer or fill in the blanks type subjective type questions with added intelligence at some extent. The examination process becomes interesting and interacting in which examinee is required to enter one word answer. The solution is obtained that will automatically evaluate, identify the answer text entered by the user and generating the correct text pattern suggestions which are phonetically nearer to the entered word only if it contains typographical mistakes.

The solution can be implemented for on-line examination involving "Fill in the blanks" or "one word question's answer". This may be quite helpful for filling up the correct answers to the students who know the correct answers but not the correct spelling of the answer which encourage the students by suggestions, reliable effective evaluation, save time of teacher's community and obtaining quick and error free result of examination.

\section{General Terms}

Online examination, computerized test,

\section{Keywords}

Automatic Evaluation, Objective and subjective tests, MCQ, One word question answer, Pattern recognition, Artificial intelligence.

\section{INTRODUCTION}

Online computerized test is popular to education system. Such examinations are prepared, organized, evaluated and result also declared by use of computer systems. Such examinations in most of the cases involve multiple choice questions (MCQs) which is preferable to assess objective kind of knowledge but in this paper other type of subjective questions like one word answering or fill in the blank type of questions without any options are approached to assess subjective type question at limited extent moving one step towards the assessment of descriptive answers which requires extraordinary efforts to design, develop and implement for variety of subjects.

The research paper describes usage of computer in different ways in educational system. One such use is to assess knowledge of a student for one word answer. A model is constructed for this concept having added artificial intelligence of text pattern recognition. That will help to students, teachers and as a result to entire society. At last the solution is concluded.

\section{USE OF COMPUTERS IN EXAMINATION PROCESS}

Many applications exists for conducting of online examinations, few of them are briefly discuss here.

2.1 Traditional hand written examination and manual evaluation and generating a result using computer.

2.2 Digital evaluation of scanned copy of answer sheets using computer connected to network through LAN or internet with suitable interface. This type of evaluation still requires the human effort and just an improvement over manual assessment .

2.3 MCQ examination is organized off line and require to fill Optical Mark Reader (OMR) sheet which then be scanned and evaluated using computer.

2.4 Similar to OMR, MCQ examination require to answer by writing appropriate option A, B, C, D. Optical Character Reader (OCR) requires to recognize written option for different hand written options and then evaluating using computer.

2.5 Improvement to this is online MCQ examination in which user require to select correct answer using radio button and evaluating the answer by comparing with the given key. This type of examination saves time and reduces the human effort with quick declarion of result. 
which a sentence is answered. This requires subjective domain knowledge capabilitiy to understand the meaning of the sentence for automatic evaluation.

2.7 Subjective type online examination and evaluation require more efforts to understand the meaning of the same answer formedin different ways to determine the level of knowledge from answer.

2.8 Descriptive type online examination evaluation of hand written answer which firstrequire to recognize the hand written answer and then require evaluating efforts with domain specific knowledge.

2.9 Evaluating the knowledge from directly asking the question and recognizing the answer using speech recognition technique.

2.10 Evaluating match the pair, true or false, arrange in specific order like questions[1][2]

2.11 Evaluation examinination involving fillin the blank or one word answer question. This pattern of examination require userto enterone word as an answer.

All this examinations have their own characteristics and advantages based on the needs of examination criteria. This paper focuses around the one word answering examination evaluation system.

\section{INTRODUCTION OF PHONETIC ALGORITHMS}

Phonetic algorithms are used to determine and compare the phonetic equality between given words. Various phonetic algorithms are developed based on needs and languages. Some popular such algorithms are described in following section[12][13].

3.1 Soundex algorithm was originally developed by Robert C. Russell and Margaret K. Odell in 1918 which returns a four character string for the given word in which the first character is the starting alphabet of the given word and remaining three are digits representing the phonetic encoding[14].

3.2 Daitch-mokotoff soundex is a variation of original soundex and named as D-M soundex which was designed in 1985 by Gary mokotoff and later improved by Randy Daitch to match surnames of Slavic and German languages and returns the six digit numeric code for the given word.

3.3 Kolner phonetic algorithm is similar to soundex but was designed for German words.

3.4 Metaphone family of algorithms are suitable for most of the English words and these algorithms are the basis for many English spell checkers and dictionaries. First metaphone algorithm was developed by Lawrence Phillips in 1990. Later variation of metaphone by him was double metaphone and incorporating other languages too. In 2009 he released the third version of metaphone which achieves accuracy of $99 \%$ of English words[15].

3.5 NYSIIS means New York state Identification and Intelligence System which is known as NYSIIS phonetic algorithms developed in 1970 which has achieved increased accuracy on soundex.

3.6 The match rating Approach (MRA) is a phonetic algorithms whichwas developed by Western Airlines in 1977 for indexing and comparing homophonous names. MRA uses distance calculation between two words.

3.7 The Caverphone phonetic algorithm was developed by David Hood at the University of Otago in New Zealand in 2002 and revised in 2004 and was created for data matching between late $19^{\text {th }}$ century and early $20^{\text {th }}$ century electoral rolls to commonly recognize the names and surnames.

All this algorithms have their own characteristics and advantages. This algorithm is used in the proposed model to search a set of words which have similar pronunciation of the given word. One or more algorithms can be used for improving process of search family of homophones for a given words and used as suggestions to the user which are phonetically neared to the given word when user makes any typographical error.

\section{INTRODUCTION PATERN RECOGNITION}

Pattern recognition is the popular research area in computer science. Number of applications exist that uses pattern recognition techniques. In pattern recognition an object is recognized using specific characteristics that uniquely describes and differentiates it from thousands of other objects. Popular techniques of pattern recognition are listed below [8][9].

4.1 Statistical Pattern recognition

4.2 Probability Density Estimation

4.3 Fuzzy set theory techniques

4.4 Neural network

4.5 machine learning and signal processing

\section{PATERN RECOGNITION APPLICATIONS}

Many computer applications use pattern recognition and few of these applications are listed below[7].

5.1 Recognizing and classifying texts related to some specific domains

5.2 Recognizing synonyms and antonyms

5.3 Recognizing and grouping homophones

5.4 Recognizing texts from the voice

5.5 Recognizing hand written texts

5.6 Recognizing texts patterns for NLP

5.7 Recognition of human face

5.8 Recognition of human thumb signature

5.9 Recognition of an object from image

5.10 Recognition specific DNA pattern

5.11 Recognition of specific products bought together

All these applications may use different techniques of pattern recognition. The present paper uses text pattern recognition to 
recognize homophones which have similar pronunciation from large amount of data and incorporating this with an online examination system designed for one word answer question and fill in blank type question. The pattern recognize as homophone family is used to help and suggest alternative answer to the user in response to any misspelled or typographical error occurs in input answer. This makes examination interacting and interesting and motivate the user experience when user knows correct pronunciation but not the correct English spelling.

\section{AUTOMATIC ONE WORD COMPUTERIZED TEST MODEL WITH INTEGRATION OFPHONETIC ALGORITHM}

One word answer questions examination can be implemented by use of phonetic algorithm. The phonetic algorithm is used when attempting answer. When user answers by entering a word it is tested with predefined list of word dictionary. We require a dictionary having only word list without meaning. This word list should be prepared first. The dictionary is used to check the correctness of the entered word. If the entered word is found in dictionary then is accepted. But may be the case, the entered word is incorrect in form of misspelled or typographic mistake, the word will not be found from the list of words. Phonetic algorithm is used to determine nearest words which has pronunciation similar to the given word. Each word is phonetically compared with the entered word using any phonetic algorithm or set of algorithm to determine its phonetic family of words. The matching list words are propagated to the user. User then can select any one word as an answer. In either case of input, the test can receive only correct word. Further improvement can be applied when any name entered as an answer then it must be compared with dictionary of names. Name list should be large enough to cover most of the general and popular names. Also same phonetic algorithm can be applied when a misspelled name is entered and list of phonetic similar name list should be displayed for selection.

The mechanism applied here in this model can be helpful to student who knows correct pronunciation but due to large vocabulary of English language enters wrong spelling word as an answer. This will motivate the user experience and makes examination interacting as well as interesting.

The behavior of the evaluation process will become natural when any examiner assesses answer sheet manually and finds spelling mistake in answer, depending on the examiner can grant the mark where subjective knowledge is given more important compared to spelling or grammatical mistakes. Thus it is computerized online test, with having artificial intelligence in evaluating the answers as well as generating and suggesting the examinee which will be beneficial to the student. Thus functional behavior of the computerized test is similar to the efforts required by an examiner to assess the answer.

The model can also be useful for preparation of the question and answer by the teacher as well. There may be chances of the misspelled incorrect word answer when the test is prepared. So if the same mechanism described above can be implemented when the test is prepared by the teacher, there is less chance of loss of marks for the students who enter the correct answers. So the model is quite useful to both for teacher to prepare the test and for student to attempt the test. By using the mechanism, the question answer set prepared will obviously becomes error free and robust.

The model is depicted in following figure 1.

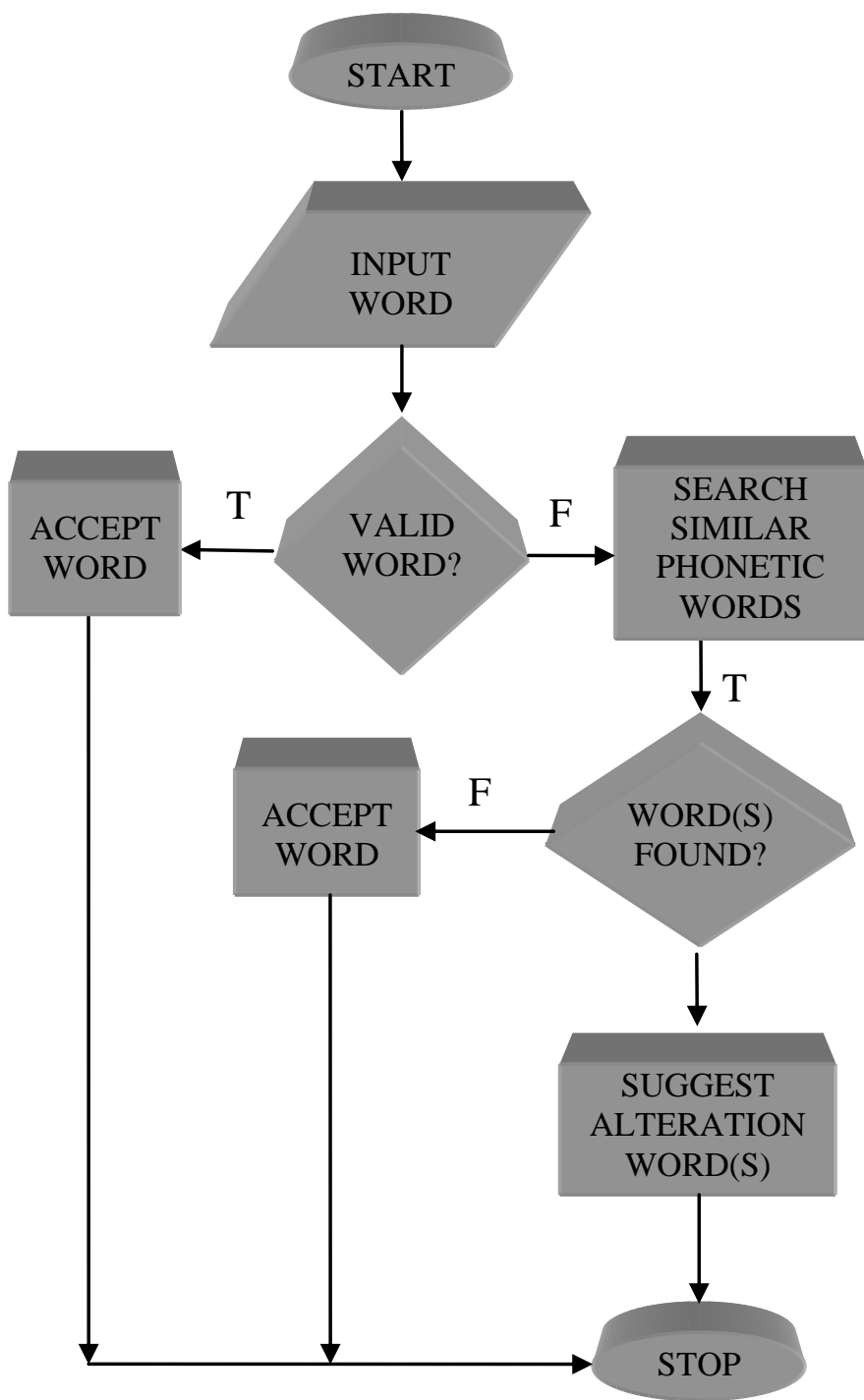

Figure 1 : One word answer computerized test model

In this model if the given word is not valid meaning that it is misspelled then alternative homophones are searched for the given word. If any word or set of words are found then alternative words are suggested to the user. But also no alternative word is found here it is assumed that the word is name and accepted as correct name. As discussed previously, same kind of processing can be applied for possible name list.

The model requires the predefined word list dictionary. Moreover name list can be extended for better performance. Also during and after the implementation of the model, the list of words should be expanded an and when found new one.

If the entered answer is valid without any typographical error, it merely accepted, and no correct answer is suggested in any case. But if invalid input is entered then the correct answer may be the part of the alternative suggestions. User must require selecting a word from list of suggested alternative answers. At last which is not included in the model an assessment process that will direct compare user responses with actual entered answer keys to prepare the result and display of marks obtained by the user. Also for this process comparison should be made by ignoring the upper case or 
lower case differences in the user answer and correct key answer.

The described model can be implemented using suitable programming language depending on needs and criteria.

\section{CONCLUSION}

The algortihm can be implemented using any programming language supporiting database and file handling capabilities. Implemetation for preparing a semantic map as per algorithm requires hours of time due to large database of words and large number of comparisons to find set of homophones, but it is just a one time processing. Once it is created searching a family of homophones becomes more efficient using both of the files compared to direct searching from large database of words.

MCQs type question answering test is now common, but to judge the subjective type knowledge of the student, this model can be considered as the first step towards the direction of subjective kind of computerized test. To implement subjective descriptive test, it requires more human efforts to design and develop and more computer processing. But the computerized test can be made more effective by combining the MCQs type question with match the pair, arranging in proper order, true or false, and one word answer type of questions.

Implemented model must be tested for desired outcome and behavior of the model. The testing of the model depends on the word list available and use of phonetic algorithms. Before incorporating the homophone matching algorithms, it should be separately tested by entering misspelled word and analyzing the suggested homophones. Same listing will be made available to the user attempting the answer with misspelled word.

So the concept discussed here in the form of model can have many future scopes and can be extended with more features to make online examination process more effective to determine knowledge level, time saving, quick result and an interesting process.

\section{REFERENCES}

[1] Automated answering for subjective examination Asmita Dhokrat, Gite Humant R., C. Namtrata Mahender - International Journal of Computer Applications (0975 - 8887) Volume 56- No.14, October 2012

[2] Assessment of Answers: Online Subjective Examination - Asmita Dhokrat, Gite Hanumant R , C.Namrata Mahender Proceedings of the Workshop on Question Answering for Complex Domains, pages 47-56, COLING 2012, Mumbai, December 2012.

[3] Automated Assessment of Short One-Line Free-Text Responses With Identifying Word Ordering Navjeet Kaur \& Kiran Jyoti - Special Issue of IJCCT, ISSN (ONLINE) : 2231-0371, ISSN (PRINT) : 0975-7449, Volume- 3, Issue-1

[4] Automated Assessment of Short One-Line Free-Text Responses In Computer Science Navjeet Kaur \& Kiran Jyoti

[5] National Online Examination System: An Architectural Perspective Pradeep Kumar, Pankaj Nirwan , P Govind Raj

[6] Computation Linguistic : Online Subjective Examination Modeling - Advances in Computational Research ISSN:
0975-3273 \& E-ISSN: 0975-9085, Volume 4, Issue 1, 2012, pp.-31-33

[7] Anatomy of pattern recognition MAYANK PARASHER, SHRUTI SHARMA, A.K SHARMA, J. P. GUPTA - Indian Journal of Computer Science and Engineering, ISSN : 0976-5166 Vol. 2 No. 3 Jun-Jul 2011

[8] Classification Techniques in Pattern RecognitionLihong Zheng and Xiangjian $\mathrm{He}$ - Conference proceedings ISBN 80-903100-8-7 WSCG'2005, January 31-February 4, 2005 Plzen, Czech Republic

[9] Pattern Recognition Techniques - Seema Asht and 2Rajeshwar Dass, International Journal of Computer Science and Telecommunications [Volume 3, Issue 8, August 2012]

[10] Analysis and Comparative Study on Phonetic Matching Techniques Rima Shah, Dheeraj Kumar Singh International Journal of Computer Applications (09758887) Volume 87 - No.9, February 2014

[11] Name and Address matching strategy - White Paper Series Truth Technologies December 2010

[12] Vimal P. Parmar, Dr. CK Kumbharana "Study Existing Various Phonetic Algorithms and Designing and Development of a working model for the New Developed Algorithm and Comparison by implementing it with Existing Algorithm(s)" International Journal of Computer Applications (IJCA) ISSN: 0975 - 8887 Volume 98 / Number 19 (ISBN: 973-93-80883-19-1) DOI : $10.5120 / 17295-7795$

[13] Phonetic algorithm meaning and description http://en.wikipedia.org/wiki/Phonetic_algorithm

[14] Soundex algorithm description and working mechanism http://en.wikipedia.org/wiki/Soundex

[15] Metaphone algorithm description and working mechanism,http://en.wikipedia.org/wiki/Metaphone

[16] Homophone and related linguistic concept https://en.wikipedia.org/wiki/Homophone

[17] Vimal P. Parmar, Apurva K. Pandya, Dr. CK Kumbharana "Determining the Character Replacement Rules and Implementing Them for Phonetic Identification of Given Words to Identify Similar Pronunciation Words" Futuristic Trends on Computation Analysis and Knowledge Management (ABLAZE) 2015 International Conference at Greater Noida, India Pages : 272-277 Print ISBN : 978-1-4799-8432-9 DOI : 10.1109/ABLAZE.2015.7155010 Publisher : IEEE

[18] Vimal P. Parmar, Dr. CK Kumbharana "Study Existing Various Phonetic Algorithms and Designing and Development of a working model for the New Developed Algorithm and Comparison by implementing it with Existing Algorithm(s)" International Journal of Computer Applications (IJCA) ISSN: 0975 - 8887 Volume 98 / Number 19 (ISBN: 973-93-80883-19-1) DOI : $10.5120 / 17295-7795$ 